Research paper

\title{
A comparison between pure active pharmaceutical ingredients and therapeutic deep eutectic solvents: Solubility and permeability studies
}

\author{
Ana Rita C. Duarte ${ }^{a, b}$, Ana Sofia D. Ferreira ${ }^{c}$, Susana Barreiros ${ }^{c}$, Eurico Cabrita ${ }^{c}$, Rui L. Reis ${ }^{a, b}$, \\ Alexandre Paiva ${ }^{\mathrm{c}, *}$ \\ a 3B's Research Group-Biomaterials, Biodegradable and Biomimetic, University of Minho, Headquarters of the European Institute of Excellence on Tissue Engineering and \\ Regenerative Medicine, Avepark 4805-017 Barco, Guimarães, Portugal \\ b ICVS/3B's PT Government Associated Laboratory, Braga/Guimarães, Portugal \\ ${ }^{\mathrm{C}}$ LAQV-REQUIMTE, Departamento de Química, Faculdade de Ciências e Tecnologia, Universidade Nova de Lisboa, 2829-516 Caparica, Portugal
}

\section{A R T I C L E I N F O}

\section{Article history:}

Received 11 October 2016

Revised 5 January 2017

Accepted in revised form 2 February 2017

Available online 9 February 2017

\section{Keywords:}

Therapeutic deep eutectic solvents

Drug solubility

Permeability

Diffusion coefficient

Pharmaceutical and biomedical applications

\begin{abstract}
A B S T R A C T
THEDES, so called therapeutic deep eutectic solvents are here defined as a mixture of two components, which at a particular molar composition become liquid at room temperature and in which one of them is an active pharmaceutical ingredient (API). In this work, THEDES based on menthol complexed with three different APIs, ibuprofen (ibu), BA (BA) and phenylacetic acid (PA), were prepared. The interactions between the components that constitute the THEDES were studied by NMR, confirming that the eutectic system is formed by H-bonds between menthol and the API. The mobility of the THEDES components was studied by PFGSE NMR spectroscopy. It was determined that the self-diffusion of the species followed the same behavior as observed previously for ionic liquids, in which the components migrate via jumping between voids in the suprastructure created by punctual thermal fluctuations. The solubility and permeability of the systems in an isotonic solution was evaluated and a comparison with the pure APIs was established through diffusion and permeability studies carried out in a Franz cell. The solubility of the APIs when in the THEDES system can be improved up to 12 fold, namely for the system containing ibu. Furthermore, for this system the permeability was calculated to be $14 \times 10^{-5} \mathrm{~cm} / \mathrm{s}$ representing a 3 fold increase in comparison with the pure API. With the exception of the systems containing PA an increase in the solubility, coupled with an increase in permeability was observed. In this work, we hence demonstrate the efficiency of THEDES as a new formulation for the enhancement of the bioavailability of APIs by changing the physical state of the molecules from a solid dosage to a liquid system.
\end{abstract}

(c) 2017 Elsevier B.V. All rights reserved.

\section{Introduction}

Pharmaceutical sciences face major challenges associated with drug solubility and permeability, which result in an inadequate pharmacokinetics and poor bioavailability of the active pharmaceutical ingredient (API) [1]. According to the biopharmaceutics classification system (BCS) the drugs can be categorized in four main groups based on their solubility and permeability [2] (Fig. 1):

This classification system is often related to the solubility in simulated body fluids and gastrointestinal permeability. Nonetheless, in vitro models have been adopted to determine the permeability of the drugs in synthetic membranes mimicking the effect of a tissue. To overcome the problem of low solubility and/or low permeability of drugs, often higher dosages are administered lead-

\footnotetext{
* Corresponding author.

E-mail address: alexandre.paiva@fct.unl.pt (A. Paiva).
}

ing to potential systemic toxicity and severe side effects. Pharmaceutical innovations require either crystal engineering strategies coupled with new methods of administration and novel delivery systems able to provide a more effective and patient compliant therapy.

Crystal engineering has been the focus of different studies which aim is to promote the enhancement of solubility and/or permeability of APIs [3]. Various methods, namely the creation of metastable polymorphs, amorphization, salt formation or cocrystal formation have been proposed in order to manipulate the APIs properties, particularly their crystallinity and consequently their bioavailability $[1,4]$. On the other hand, the manipulation of the physical and/or morphological properties of the API itself, for instance micronization of the powders may also contribute to the enhancement of drug dissolution and permeability [5]. Other strategies involve the preparation of more complex systems, such as suitable delivery systems and the design of new carriers [6-8]. 


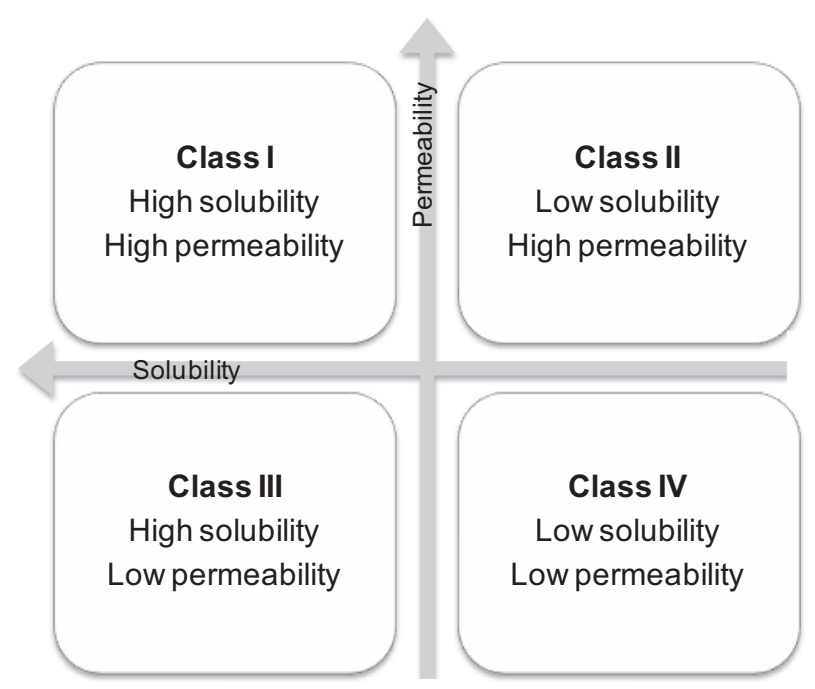

Fig. 1. Biopharmaceutics classification system of APIs.

Cherukuvada and coworkers reviewed the potential use of eutectics as improved pharmaceutical formulations [9]. However, up to date limited information has been published or reported for these systems, which are often mistaken for unstable cocrystals. The major difference between eutectics and cocrystals relies on the interactions and the type of components present in the system. An eutectic system is a mixture of two components which interact trough hydrogen bonding and lead to an overall decrease in the melting temperature of the system. When the decrease in the melting temperature is such that the systems become liquid at room temperature they are so-called deep eutectic solvents (DES). THEDES - therapeutic deep eutectic solvents have, hence, been defined as a bioactive eutectic system containing an API as one of the DES components [10]. Stott and coworkers have described for the first time a THEDES system composed of menthol and ibuprofen (Ibu) [11]. The main purpose was the enhancement of skin permeation and development of transdermal delivery systems. Similarly, Morrison and coworkers reported a 5-22,000fold increase on the solubility of BA (BA), danazol, griseofulvin and itraconazole in urea-choline chloride and malonic acidcholine chloride DES [12]. Wang et al. have reported the development of an eutectic system of lidocaine:ibu. The results of this study highlight the differences between the dissolution and permeation of the active compounds, which can be finely tuned towards the development of transdermal delivery systems [13].

The design of new THEDES systems is not easy and lack of knowledge on the type of interactions and associations established between the molecules which constitute the THEDES is still hindering the development of new systems. Nonetheless, this approach is, due to its simplicity, of utmost relevance in the pharmaceutical field. The preparation of the THEDES systems yields a $100 \%$ pure product, with no losses during production and no need for subsequent purification steps [14]. Furthermore, the scale-up of the process is relatively straightforward, which is highly important for the pharmaceutical industry [15].

In a previous work we have reported the development of novel THEDES based on choline chloride and menthol, combined with three APIs, namely acetylsalicylic acid, BA and phenylacetic acid (PA) [16]. It was herein demonstrated that the dissolution rate of the APIs is greatly enhanced by these systems, particularly in the case of menthol-based THEDES. The interest in terpenes and particularly in menthol is mostly due to its effectiveness as permeation enhancer, which has been reported in the literature [17]. The characterization of the systems in terms of dissolution rate, solubility and permeability are most relevant to the determination of the suitable dosage and form of administration. For this reason, in this work, we have further explored the menthol-based systems: menthol:ibu; menthol:BA and menthol:PA and evaluated the solubility and permeability of the APIs present in the systems. In the particular case of the systems studied the envisaged route of administration is the transdermal way. The systems prepared could be embedded in a patch, providing a more effective delivery of the API in a local delivery approach.

\section{Materials and methods}

\subsection{Materials}

The reagents used in the preparation of THEDES were menthol (99\% purity, CAS $89-78-1$, Sigma), ibu ( $>98 \%$ purity, CAS $15687-$ 27-1, Sigma), BA (>99.5\% purity, CAS $65-85-0$, Sigma) and PA (99\% purity, CAS 103-82-2, Sigma). Fig. 2 presents the chemical formulas of the reagents used. All chemicals were used without any further purification.

Phosphate buffered saline (PBS) was prepared from phosphate buffered saline tablets (Sigma), as indicated. One tablet was dissolved in $200 \mathrm{~mL}$ of deionized water, yielding a $0.01 \mathrm{M}$ phosphate buffer, $0.0027 \mathrm{M}$ potassium chloride, $0.137 \mathrm{M}$ sodium chloride, $\mathrm{pH} 7.4$ solution, at $25^{\circ} \mathrm{C}$.

\subsection{THEDES preparation}

THEDES systems prepared were menthol:Ibu (3:1); menthol:BA (3:1); menthol:PA (2:1) and menthol:PA (3:1). The systems were prepared by mixing the two components at the given molar ratio [16]. The mixture was heated to $40^{\circ} \mathrm{C}$, under constant stirring, until a clear liquid solution was formed. Typically the liquid solution was obtained after a few hours.

\subsection{Characterization}

\subsubsection{NMR studies}

All the NMR experiments were carried out in a Bruker Avance 400 spectrometer, equipped with a BBO probe capable of producing magnetic field gradient pulses up to $53.5 \mathrm{G} \mathrm{cm}^{-1}$ in the $z$ direction, with a BCUextreme temperature control unit. The THEDES samples were placed in a $5 \mathrm{~mm}$ NMR tube and left for $15 \mathrm{~min}$ at the desired temperature $\left( \pm 0.1^{\circ} \mathrm{C}\right)$ in order to reach thermal equilibrium. All chemical shifts were referred using tetramethylsilane (TMS) as internal reference and the shim was performed using a sealed C6D6 capillary tube.

\subsubsection{Pulsed-field gradient ( $P F G) N M R$}

The diffusion measurements were performed using the stimulated echo sequence using bipolar sine gradient pulses and eddy current delay before the detection [18]. For each PFGSE experiment 16 spectra of $64 \mathrm{~K}$ data points were collected, with a relaxation delay of $2 \mathrm{~s}$, in which the duration of the magnetic field gradient $(\tau)$ was 2-4 ms, the diffusion times was $80 \mathrm{~ms}$ (for the diluted samples) and $800 \mathrm{~ms}$ (for the neat samples) and the gradient recovery was $200 \mu \mathrm{s}$. The sine shaped gradient $(g)$ was incremented from 5 to $95 \%$ of the maximum gradient strength in a linear ramp (2.68$50.83 \mathrm{G} \mathrm{cm}^{-1}$ ).

To determine the self-diffusion coefficients, the spectra were first processed in the F2 dimension by standard Fourier transform and baseline correction with the Bruker Topspin software package (version 3.2). The self-diffusion coefficients were calculated by exponential fitting of the data belonging to individual columns of the $2 \mathrm{D}$ matrix using the Origin 9.0 data software program. In a PFG NMR experiment the spin echo attenuation decay, $I$, is related 
<smiles>CC1CCC(C(C)C)C(O)C1</smiles>

menthol<smiles>O=C(O)c1ccccc1</smiles>

benzoic acid<smiles>CC(C)Cc1ccc(C(C)C(=O)O)cc1</smiles>

ibuprofen<smiles>O=C(O)Cc1ccccc1</smiles>

Phenylacetic acid

Fig. 2. Chemical structure of the components used to prepare the therapeutic deep eutectic solvents.

to the experimental parameters by the Stejskal-Tanner equation (Eq. (1)) [19].

$\frac{I}{I_{0}}=e^{-q^{2} D\left(\Delta-\frac{\tau}{3}\right)}$

in which $D$ is the self-diffusion coefficient, $\Delta$ is the diffusion time, and $q$ is the gradient used in the spatial encoding and decoding of the spin, $q=\tau \gamma g$, which is a function of the gyromagnetic ratio $(\gamma)$, the gradient strength $(g)$ and the duration over which the gradient is applied $(\tau)$. The gradient coil constant was previously determined by performing a relative calibration, which implies measuring the self-diffusion coefficient of a reference substance of known diffusion (deuterated water 99.9\%). The diffusion of deuterated water was measured in a $5 \mathrm{~mm}$ NMR tube, at $\Delta=100 \mathrm{~ms}$ and $\delta / 2=0.8 \mathrm{~ms}$, fixing the self-diffusion coefficient of $\mathrm{D}_{2} \mathrm{O}$ at $1.95 \times 10^{-9} \mathrm{~m}^{2} \mathrm{~s}^{-1}$ and applying Eq. (1) the gradient coil constant used in the diffusion coefficients determination was calculated $\left(53.5 \mathrm{G} \mathrm{cm}^{-1}\right)$.

\subsubsection{Viscosity}

The viscosity of the different systems was characterized on a Kinexus Prot Rheometer (Kinexus Prot, MAL1097376, Malvern) fitted with parallel plate geometry with $20 \mathrm{~mm}$ diameter (PU20 SR1740 SS) and $1 \mathrm{~mm}$ gap. The steady-state flow measurements were performed under controlled-stress conditions and a shear rate of $1 \mathrm{~s}^{-1}$ at $20,25,30,35,40,45$ and $50^{\circ} \mathrm{C}$. The data is represent as the average of ten measurements.

\subsection{Solubility measurements}

The solubility measurements were performed with the pure APIs and the respective THEDES. Briefly, an excess of API and THEDES was added to PBS solution at room temperature $\left(20^{\circ} \mathrm{C}\right)$ and stirred for $24 \mathrm{~h}$. The determination of the API solubility was performed by UV spectroscopy in a microplate reader (BIO-TEK, SYNERGY HT). The absorbance of the solutions was measured at the API maximum absorption wavelength, respectively, at $265 \mathrm{~nm}$ (ibu), $290 \mathrm{~nm}$ (BA) and $230 \mathrm{~nm}$ (PA). A calibration curve using the respective THEDES or the API as standards was prepared for quantification.

\subsection{Permeability studies}

Permeability measurements were conducted using a glass Franz-type diffusion cell (PermeGear) with a $8 \mathrm{~mL}$ reactor compartment with an effective mass transfer area of $1 \mathrm{~cm}^{2}$. The membrane used was a polyethersulphone (PES-U) membrane, with $150 \mu \mathrm{m}$ thickness and $0.45 \mu \mathrm{m}$ pore size (Sartorius Stedim Biotech, Germany), which was placed between the two compartments and held with a stainless steel clamp. The receptor compartment was filled with PBS solution, and air bubbles were removed. Afterwards, the donor compartment was loaded with the same amount of API in the powder or in the THEDES form and $2 \mathrm{~mL}$ of PBS were added. A concentration of the API in the donor compartment of $2-2.5 \mathrm{mg} /$ $\mathrm{mL}$ was obtained. Aliquots of $200 \mu \mathrm{L}$ were withdrawn from the receptor compartment at predetermined time periods (5 min and hourly from 1 to $8 \mathrm{~h}$ ) and fresh PBS was added to complete the volume. The experiments were performed at $37^{\circ} \mathrm{C}$, and the receptor compartment was stirred at $300 \mathrm{rpm}$ using a magnetic bar to eliminate the boundary layer effect.

The determination of the API diffused was performed by UV spectroscopy in a microplate reader (BIO-TEK, SYNERGY HT), according to what was previously described for the solubility studies. The cumulative mass of drug diffused to the receptor compartment was determined taking into consideration the replacement of aliquots with fresh medium and the dilution derived from the addition of fresh buffer.

The permeability, $P$ of the APIs through the membrane was calculated according to Eq. (2):

$-\ln \left(1-\frac{2 C_{t}}{C_{0}}\right)=\frac{2 A}{V} \cdot P \cdot t$

where $C_{t}$ is the concentration in the receptor compartment at time $t$, $C_{0}$ is the initial concentration in the donor compartment, $A$ is the effective mass transfer area, $V$ is the total volume of solution in both compartments [20,21].

According to a derived solution of Fick's law of diffusion it is possible to determine the diffusion coefficient, $D$ of the API through the membrane [22]. Eq. (3), was hence applied to the different systems:

$D=\frac{V_{1} \cdot V_{2}}{V_{1}+V_{2}} \cdot \frac{h}{A} \cdot \frac{1}{t} \ln \left(\frac{C_{f}-C_{i}}{C_{f}-C_{t}}\right)$ 
where $C_{f}$ and $C_{i}$ are the final and initial concentration in the receptor compartment and $C_{t}$ is the concentration in the receptor compartment at time $t$. $V_{1}$ and $V_{2}$ are the volume in the donor and in the receptor compartment respectively and $h$ is the thickness of the membrane.

\section{Results and discussion}

Solubility and permeability experiments were carried out for the pure API and for the API when part of a THEDES. To better understand the solubility and permeability behavior of the API, the interactions between menthol and the API that form the THEDES and the self-diffusion coefficients of the individual components were studied by NMR spectroscopy.

\subsection{NMR studies}

The menthol:API THEDES were analyzed by NMR spectroscopy. Fig. 3 shows the ${ }^{1} \mathrm{H}$ NMR spectrum of menthol:PA (3:1) at $298 \mathrm{~K}$, immediately after formation of the liquid mixture, with the resonances of the two THEDES components referred in the spectrum. All the THEDES ${ }^{1} \mathrm{H}$ NMR spectra show sharp and well defined chemical shift signals, with the OH protons of both THEDES components corresponding to only one sharp chemical shift $\left(\delta_{\mathrm{OH}}\right)$ at low field (vide supplementary materials). The presence of only one resonance attributed to both hydroxyl groups of menthol and carboxylic acid strongly indicates that the both types of protons are in fast exchange in the NMR timescale. The same interaction was also observed for menthol:BA (3:1) and for menthol:Ibu (3:1).

The system menthol:PA presented however an unusual behavior, as differences in the spectra were observed with time. A new set of NMR signals (4.74 ppm, td, $10.7 \mathrm{~Hz}, 4 \mathrm{~Hz} ; 0.75 \mathrm{ppm}, \mathrm{d}$, $6.8 \mathrm{~Hz}$ ) was observed $3 \mathrm{~h}$ after the formation of the system men-
thol:PA (2:1) and $24 \mathrm{~h}$ after the formation menthol:PA (3:1) suggesting that these systems were not stable. These signals were identified as the esterification product between menthol and PA. The esterification reaction conventionally takes place in the presence of an activator (acid or base catalyst), or at high temperatures. For the studied THEDES systems, even at higher temperatures ( $323 \mathrm{~K}$ ), only the menthol:PA showed the ester formation. It was also observed that after 1 week the reactions where in equilibrium with a esterification extent of $15 \mathrm{~mol} \%$ for menthol:PA (2:1) and 6 mol\% for menthol:PA (3:1). All further experiments with these THEDES were only performed after the system was in equilibrium with no change in its properties. It is important to notice that even with the presence of a third component the eutectic system was liquid at room temperature. The dependence on the molar ratio of menthol to PA strongly suggests that the reaction occurs due to the fact that PA acts both as reagent and catalyst. The higher reactivity towards esterification of PA relative to the other APIs can be explained by the influence of the substituents adjacent to the carboxylic group that have a retarding effect. In the case of $\mathrm{BA}$, the phenyl group and the carboxylic group are conjugated, which lowers the initial energy of the BA relative to PA.

The formation and stability of THEDES liquid structure can be associated to the formation of hydrogen bonds between its components. To further understand the dynamics of the hydrogen bonding in the THEDES we collected ${ }^{1} \mathrm{H}$ NMR spectrum in a temperature range of $298-323 \mathrm{~K}$. Only the systems with a molar ratio of 3:1 were studied which, in the case of PA, had a higher stability, showing no ester formation during the temperature variation studies. Temperature variation studies have been one of the most used approaches to study not only the presence of H-bonds, but also their nature and strength. The chemical shift dependence on the temperature for $\mathrm{H}$-bonded protons (both intra and intermolecular) has been reported in the literature $[23,24]$ and in the case of proteins and peptide it has been used for conformational analysis [25]. Usually, an increase in temperature was translated into an

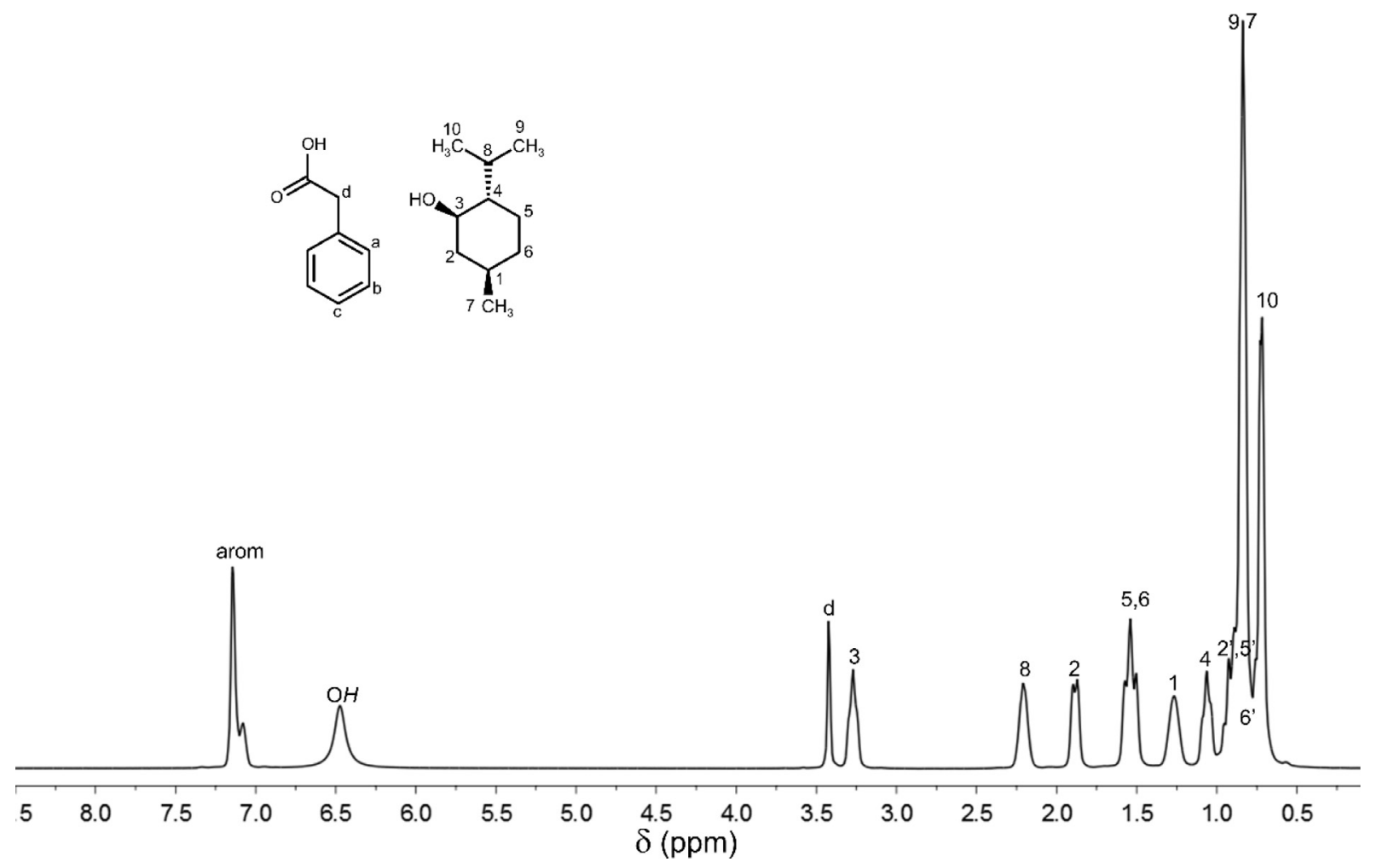

Fig. 3. ${ }^{1} \mathrm{H}$ NMR spectrum of neat menthol:PA (3:1) at $298 \mathrm{~K}$. All the resonances are attributed. 
upfield shift (smaller ppm) of the H-bond chemical shifts, due to a decrease of the intermolecular hydrogen bonding (Fig. 4a) and supplementary material). Its magnitude can be quantified by the temperature coefficient (TC). TC was obtained from the slope of the plot of the chemical shift versus temperature. As depicted in Fig. 4b), the chemical shift versus temperature shows a linear correlation, with very similar TC values for the studied systems (ca. $\left.-19 \mathrm{ppb} \mathrm{K}^{-1}\right)$. TC values more negative than $-8 \mathrm{ppb} \mathrm{K}^{-1}$ are related to large conformational changes with the temperature which is associated to intermolecular hydrogen bonding [26]. The chemical shift of a nucleus is the resonance frequency of the nucleus relative to a standard at a given temperature, and hence can be energy related. Therefore the TC gives information about the strength of the hydrogen bond, i.e. the energy changes of the H-bond. Analyzing the effect of the carboxylic acid in the $(3: 1)$ THEDES it is observe that the hydrogen bonds are independent on the API, at all studied temperatures, and apparently of the same type (intermolecular bonding) and similar energy, which indicates an analogous global structure.

One of the approaches used towards the understanding of the macroscopic behavior of the THEDES was by studying the transport properties of the different components of the THEDES mixture, which gave an insight to the interactions present in these systems. PFG NMR allowed the determination of the self-diffusion coefficients, which are random thermal translational motion of molecules or ions under conditions of thermodynamic equilibrium. The advantage of PFG-NMR is that it allowed the simultaneously determination of the self-diffusion coefficients of the different components of the THEDES, allowing to discriminate between different chemical moieties of the components.

Since all the THEDES studied in this work are menthol based and have the same menthol:API molar ratio, the differences in transport properties can be stated to be mostly related to the interactions between the two components.

A typical spin echo attenuation of the PFGSE NMR measurements at different temperatures is shown in Fig. 5.

The logarithm of the spin echo decay of all the THEDES resonances shows a linear correlation with $q^{2}(\Delta-\delta / 3)$ (Eq. (1)), as expected for a homogeneous sample. The self-diffusion coefficients for both components were obtained from the slope of the StejskalTanner equation (Eq. (1)) in the logarithmic form, i.e., logarithm of $I$ vs $q^{2}(\Delta-\delta / 3)$ plot (Table 1$)$.
The self-diffusion coefficients of the API are lower than those of the menthol, independent of the molecular dimension of the API and the range of temperatures. Carboxylic acids exist in solution either in the ionic form, with the negative charge delocalized over the carboxylate group or as dimeric associations. The dimerization phenomena can explain the lower mobility of the API, in which the API forms a higher molecular size species, a dimer, which shows a lower self-diffusion coefficient.

A similar behavior has been reported by D'Agostino et al. [27] for maline, a NADES formed by choline chloride and malonic acid (1:1 molecular ratio) in which the authors stated that due to the dicarboxylic moieties of the malonic acid it would form chains between the malonic acid molecules, that would create constraints for the choline chloride motion that would reflect a lower selfdiffusion coefficient for the malonic acid.

The self-diffusion coefficients present, at lower temperatures, similar values for both THEDES components that, as temperature increases, start to deviate from each other due to the weakening of the chemical interaction between the two components.

The self-diffusion dependence on the temperature can be fitted by a linearization of the Arrhenius equation (Eq. (4)) in order to determine the activation energy $\left(E_{D}\right)$ correspondent to the changes in the self-diffusion due to the temperature gradient.

$\ln D=\ln D_{0}-\frac{E_{D}}{R T}$

where $T$ is the temperature $(\mathrm{K}), D_{0}$ is a constant $\left(\mathrm{m}^{2} \mathrm{~s}^{-1}\right), E_{D}$ is the activation energy $\left(\mathrm{kJ} \mathrm{mol}^{-1}\right)$ and $R$ is the gas constant $\left(\mathrm{kJ} \mathrm{K}^{-1} \mathrm{~mol}^{-1}\right)$.

The Arrhenius model shows good agreement with the experimental data, with $R^{2}$ superior to 0.99 . In Table 2, are summarized the activation energies determined for both components in each NADES

Globally the activation energies determined from the selfdiffusion coefficients are in agreement with the viscosity data, with the higher values of energy - menthol:Ibu (3:1) - corresponding to the THEDES with higher viscosity. The diffusional activation energies obtained for the menthol component are higher than the carboxylic acid in the menthol:BA (3:1) and menthol:PA (3:1), which suggests that menthol mobility has a stronger dependence on temperature. This is not the case for the menthol:Ibu (3:1), in which the diffusional energy of Ibu is higher than menthol, indicating that a)

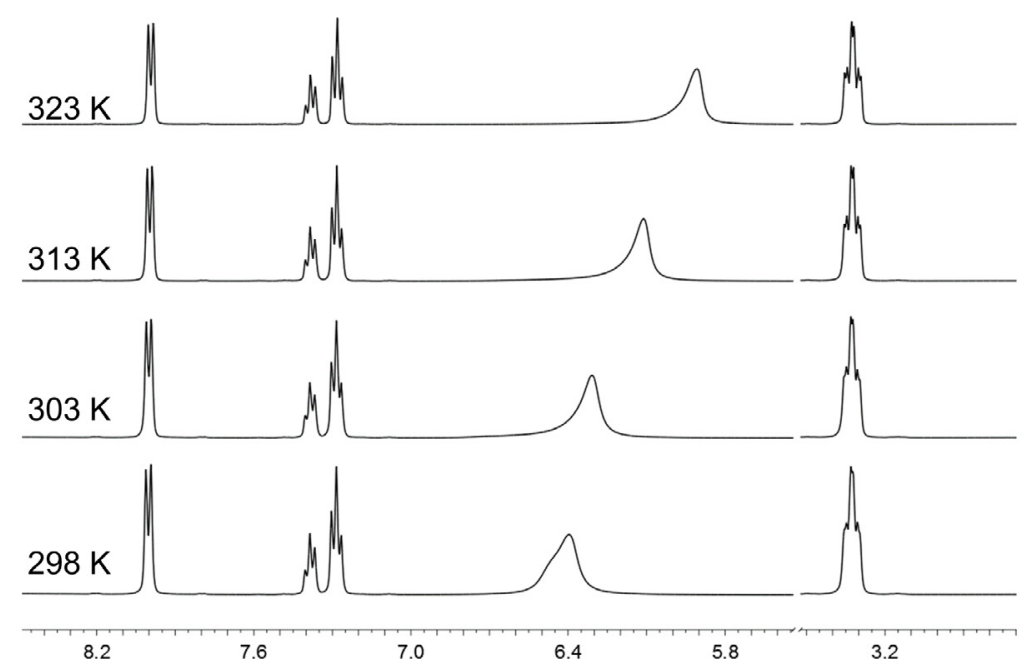

b)

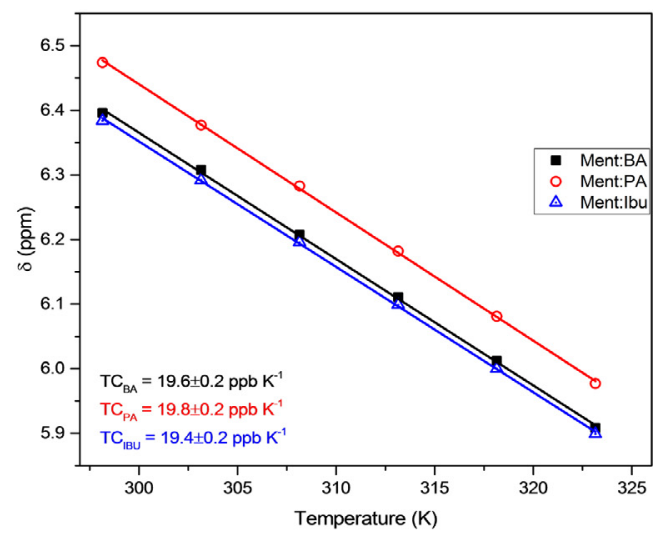

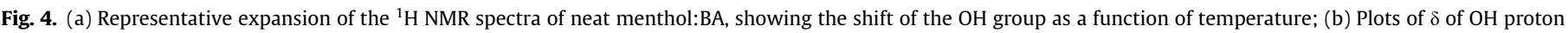
versus temperature for the studied THEDES. 
a)

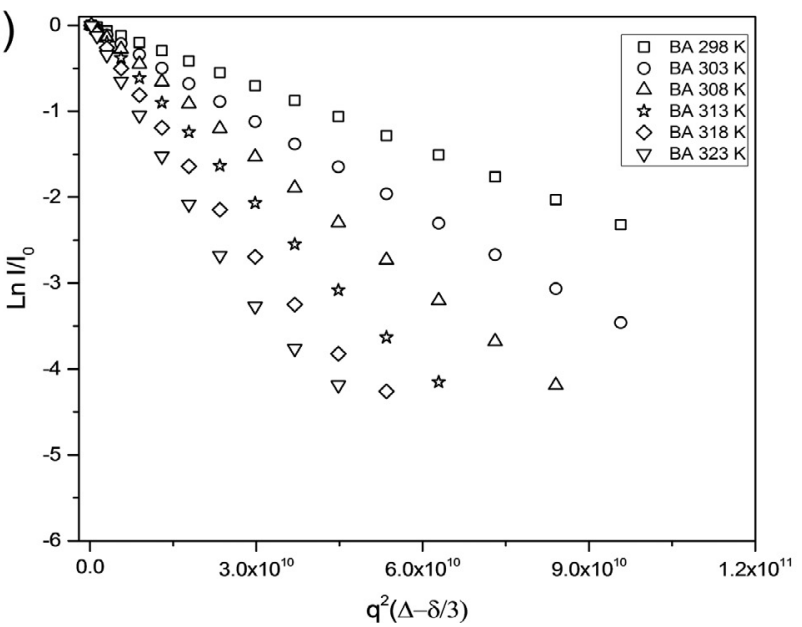

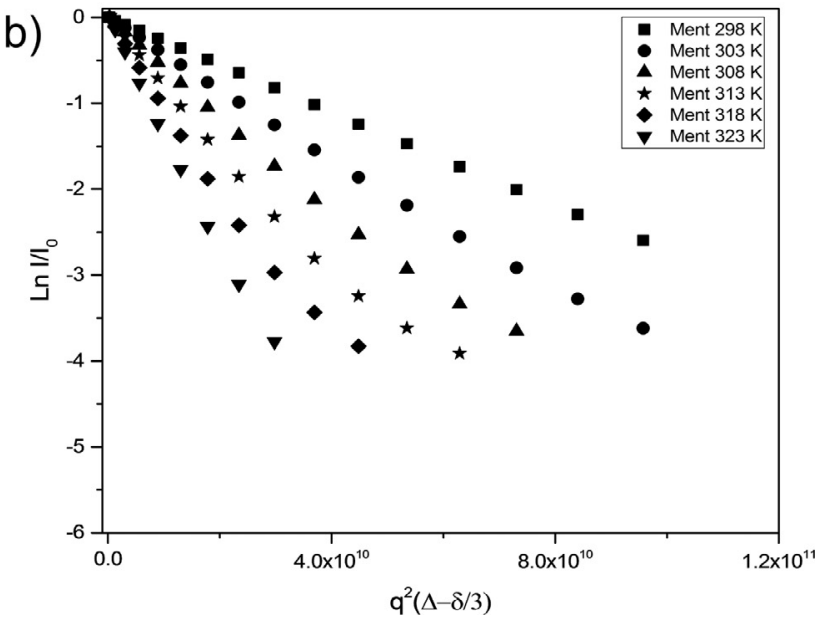

Fig. 5. Logarithm of the spin echo attenuation $v s q^{2}(\Delta-\mathrm{i} / 3)$ at different temperatures for the Menthol:BA (3:1): (a) menthol and (b) BA component.

Table 1

Self-diffusion coefficients and viscosity data for the THEDES presented in this study at different temperatures.

\begin{tabular}{|c|c|c|c|c|c|c|c|c|c|}
\hline \multirow[b]{2}{*}{$T(\mathrm{~K})$} & \multicolumn{3}{|c|}{ Menthol:Ibu (3:1) } & \multicolumn{3}{|c|}{ Menthol:PA (3:1) } & \multicolumn{3}{|c|}{ Menthol:BA (3:1) } \\
\hline & $\eta(\mathrm{mPa} \mathrm{s})$ & $\begin{array}{l}\times 10^{-11} D_{\text {Ment }} \\
\left(\mathrm{m}^{2} \mathrm{~s}^{-1}\right)\end{array}$ & $\begin{array}{l}\times 10^{-11} D_{\text {API }} \\
\left(\mathrm{m}^{2} \mathrm{~s}^{-1}\right)\end{array}$ & $\eta(\mathrm{mPa} \mathrm{s})$ & $\begin{array}{l}\times 10^{-11} D_{\text {Ment }} \\
\left(\mathrm{m}^{2} \mathrm{~s}^{-1}\right)\end{array}$ & $\begin{array}{l}\times 10^{-11} D_{\text {API }} \\
\left(\mathrm{m}^{2} \mathrm{~s}^{-1}\right)\end{array}$ & $\eta(\mathrm{mPa} s)$ & $\begin{array}{l}\times 10^{-11} D_{\text {Ment }} \\
\left(\mathrm{m}^{2} \mathrm{~s}^{-1}\right)\end{array}$ & $\begin{array}{l}\times 10^{-11} D_{\mathrm{API}} \\
\left(\mathrm{m}^{2} \mathrm{~s}^{-1}\right)\end{array}$ \\
\hline 298 & 80.23 & 1.74 & 1.15 & 30.27 & 3.57 & 3.14 & 44.15 & 2.77 & 2.34 \\
\hline 303 & 64.57 & 2.66 & 1.73 & 27.58 & 5.00 & 4.41 & 37.49 & 4.21 & 3.85 \\
\hline 308 & 52.44 & 4.20 & 2.54 & 24.89 & 6.86 & 5.99 & 32.09 & 5.85 & 5.08 \\
\hline 313 & 44.5 & 5.74 & 3.54 & 23.16 & 9.16 & 7.85 & 29.43 & 7.91 & 6.88 \\
\hline 318 & 38.05 & 7.74 & 4.88 & 21.08 & 12.06 & 10.28 & 26.06 & 10.11 & 9.06 \\
\hline 323 & 33.20 & 9.45 & 6.34 & 19.85 & 16.54 & 14.10 & 24.08 & 13.44 & 11.64 \\
\hline
\end{tabular}

Table 2

Diffusional activation energies of the THEDES components.

\begin{tabular}{lll}
\hline & \multicolumn{2}{l}{ Activation energy $\left(\mathrm{kJ} \mathrm{mol}^{-1}\right)$} \\
\cline { 2 - 3 } & Menthol & API \\
\hline Menthol:Ibu (3:1) & 53.5 & 54.9 \\
Menthol:BA (3:1) & 50.3 & 49.7 \\
Menthol:PA (3:1) & 48.5 & 47.5 \\
\hline
\end{tabular}

the mobility of Ibu requires higher energy than the menthol and the other carboxylic acids. This can suggest that Ibu is in a dimeric form.

Having determined the viscosity of the systems, it is possible to evaluate if the diffusion mechanism observed for the THEDES studied obeys the Stokes-Einstein equation (Eq. (5))

$D=\frac{k T}{c \pi \eta r_{S}}$

where $k$ is the Boltzmann constant, $T$ the absolute temperature, $c$ a constant, $r_{S}$ the hydrodynamic (Stokes) radius.

If a linear correlation is obtained between the diffusion coefficients and the $T \eta^{-1}$, the diffusion mechanism obeys the StokesEinstein equation. In our systems we observe, however, a clear deviation from linearity (Fig. 6), which suggests that the diffusion mechanism cannot be explained by the classical theory. This deviation in the self-diffusion in THEDES has already been observed and reported for NADES derived from choline chloride and it has been proposed that the diffusion mechanism in the NADES must be similar to the mechanism in ionic liquids, in which a species migrates via jumping between voids or holes in the liquid created by punctual thermal fluctuations $[27,28]$. The studied THEDES although having a molecular component behave like an ionic liquid

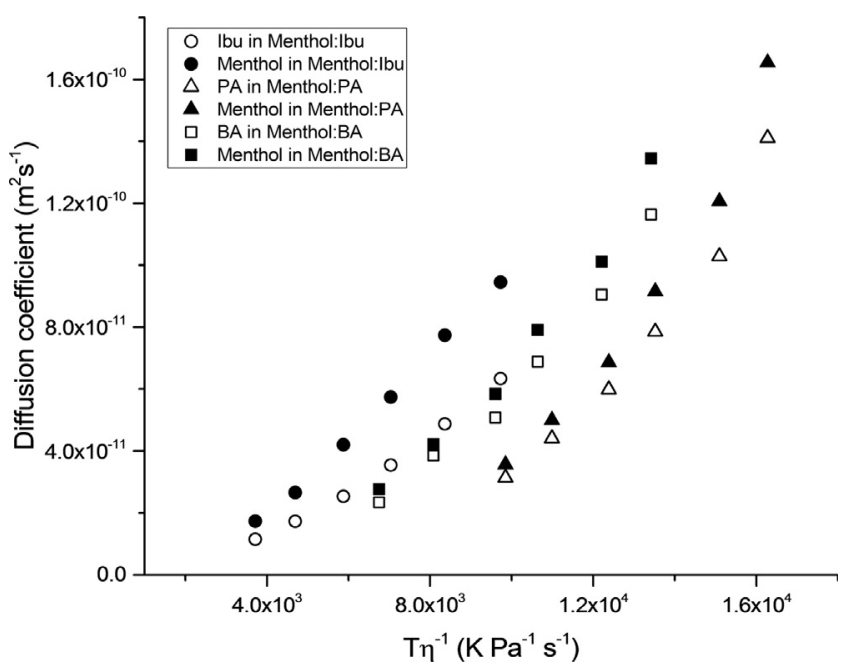

Fig. 6. Plot of the diffusion coefficients $v s \mathrm{T \eta}^{-1}$.

with strong ionic/electrostatic interactions, in which the classical diffusion theory does not apply.

\subsection{Solubility studies}

The complexation of APIs with another component in the form of a THEDES resulted in changes in the dissolution rate of the API, as described in our previous work [16]. However it has not been discussed the solubility of the API in the isotonic solution. Table 3 presents the solubility of the different APIs studied in this work together with the solubility of the API when in the liquid THEDES form. 
Table 3

Solubility of APIs in powder form or complexed in THEDES in PBS solution $\mathrm{pH} 7.4$.

\begin{tabular}{ll}
\hline & Solubility $(\mathrm{mg} / \mathrm{mL})$ \\
\hline Ibu & $2.1 \pm 0.23$ \\
Menthol:ibu (3:1) & $26.8 \pm 2.62$ \\
BA & $105.0 \pm 11.85$ \\
Menthol:BA (3:1) & $218.7 \pm 21.71$ \\
PA & $18.4 \pm 2.13$ \\
Menthol:PA (2:1) & $22.5 \pm 2.38$ \\
Menthol:PA (3:1) & $16.1 \pm 1.90$ \\
\hline
\end{tabular}

As it can be observed not all the systems present the same behavior. The most evident increase in solubility is observed for Ibu, which presents a 12 fold increase in the solubility from $2.1 \mathrm{mg} / \mathrm{mL}$ to $26.8 \mathrm{mg} / \mathrm{mL}$. In the case of the system composed of BA the solubility of the API increases 2 fold. However, for the systems containing PA the solubility only shows a slight increase when in the form menthol:PA 2:1. Furthermore, the system menthol:PA 3:1 does not present any significant differences when compared with the pure API. The formation of the ester in these THEDES may result in a change in polarity and even in the intermolecular interactions explaining the behavior observed.

\subsection{Permeability studies}

Permeability and diffusion of the APIs in aqueous systems may be changed when the compounds are combined in THEDES. $\mathrm{Ng}$ and co-workers presented a systematic study of transmembrane diffu-
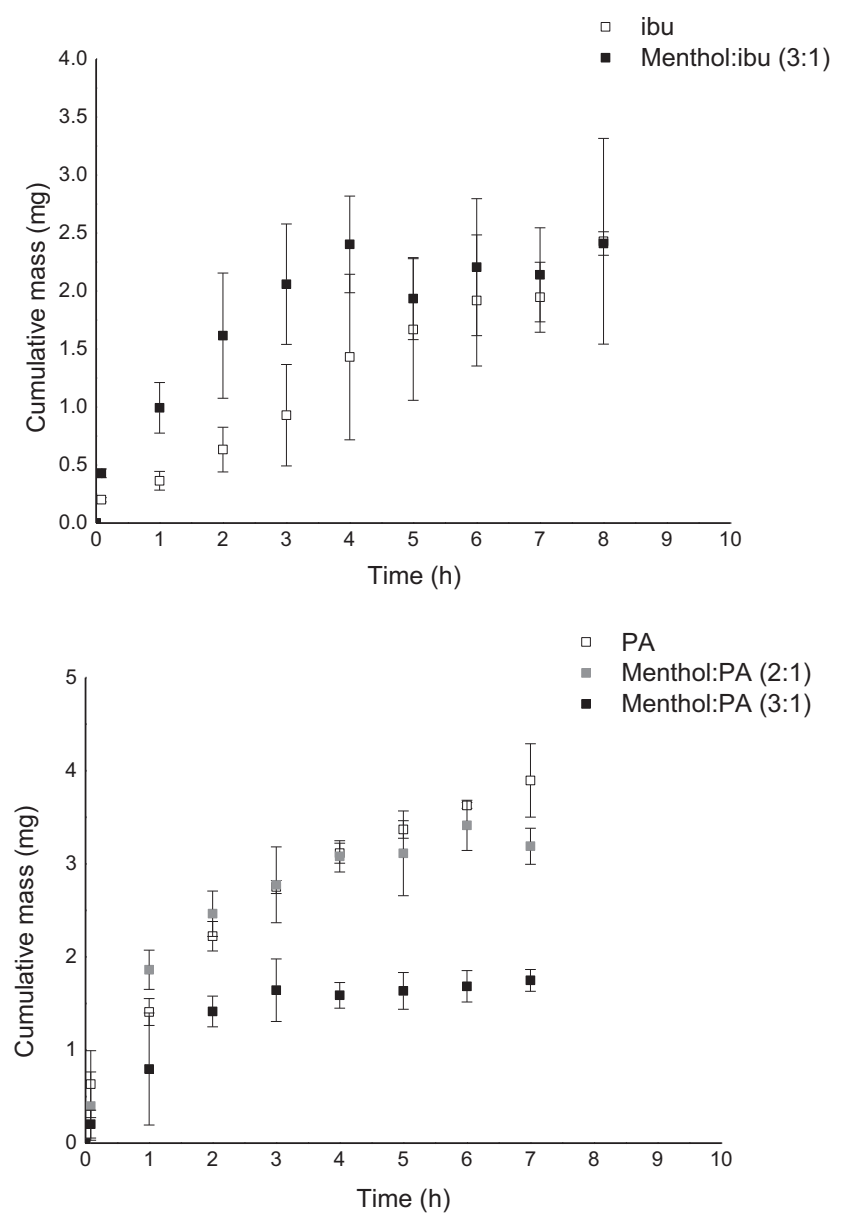

sion of Ibu through a series of different synthetic polymers and have found that considerable differences may be observed depending on the properties of the membrane itself [29,30]. In this work, we have studied the permeability using a commercially available polyethersulfone membrane. The diffusion kinetics of the each API and THEDES system are presented in Fig. 7.

Different diffusion profiles were obtained for the systems studied. While the diffusion is much higher in the case of the THEDES systems containing Ibu and BA, the diffusion of PA is only slightly enhanced when the API is in the THEDES form. For Menthol:PA (3:1), the equilibrium is reached at a lower concentration that for the pure API. As observed before menthol reacts with PA forming an ester. Although the mixture remains liquid at room temperature and continuous to behave as a THEDES, the formation of this new

Table 4

Time necessary for $50 \%$ of the API to diffuse through the membrane.

\begin{tabular}{ll}
\hline & Time $(\mathrm{h})$ \\
\hline Ibu & $>8$ \\
Menthol:ibu (3:1) & 3 \\
BA & 3.5 \\
Menthol:BA (3:1) & 0.5 \\
PA & 2 \\
Menthol:PA (2:1) & 2 \\
Menthol:PA (3:1) & $3^{\mathrm{a}}$
\end{tabular}

a The final concentration of the API in the receptor vessel is lower that the pure API.

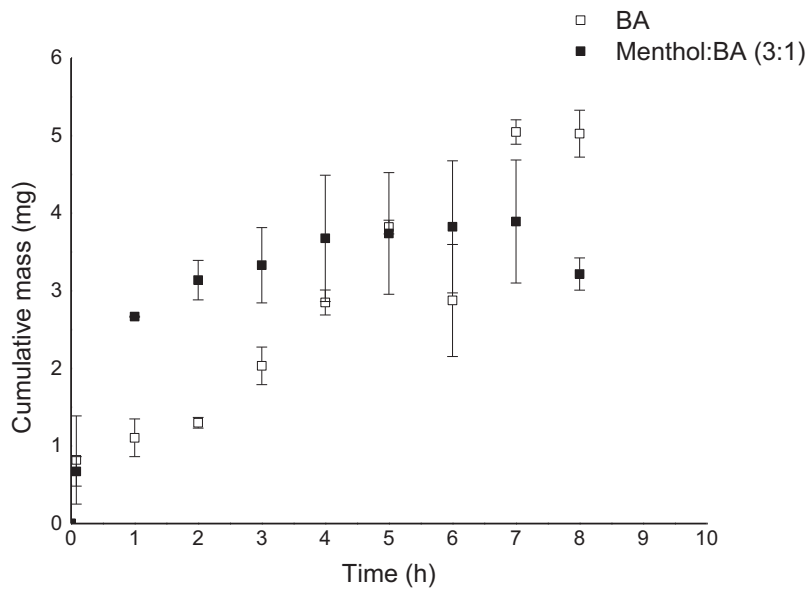

Fig. 7. Diffusion curves of the APIs and THEDES systems through a PES membrane in PBS solution (pH 7.4). 
Table 5

Permeability and diffusion coefficient calculated for the different systems.

\begin{tabular}{lll}
\hline & $\begin{array}{l}\text { Permeability } \\
\left(10^{5} \mathrm{~cm} \mathrm{~s}^{-1}\right)\end{array}$ & $\begin{array}{l}\text { Diffusion coefficient } \\
\left(10^{6} \mathrm{~cm}^{2} \mathrm{~s}^{-1}\right)\end{array}$ \\
\hline Ibu & $4.6 \pm 0.14$ & $2.39 \pm 0.43$ \\
Menthol:ibu (3:1) & $14 \pm 1.53$ & $4.32 \pm 0.34$ \\
BA & $0.9 \pm 0.01$ & $0.73 \pm 0.09$ \\
Menthol:BA (3:1) & $6.8 \pm 0.63$ & $6.43 \pm 1.42$ \\
PA & $16 \pm 2.3$ & $3.14 \pm 0.18$ \\
Menthol:PA (2:1) & $18 \pm 0.38$ & $5.73 \pm 0.43$ \\
Menthol:PA (3:1) & $13 \pm 0.59$ & $4.66 \pm 0.13$ \\
\hline
\end{tabular}

component can explain the differences observed in the diffusion curves.

As a comparative measure we estimated the time for $50 \%$ of the drug to be diffused through the membrane, and the results are presented in Table 4.

In the case of Ibu and BA the changes in the diffusion through the membrane, when the system is in the THEDES form are highly noticeable. The system menthol:PA also does not present any major differences when compared to the powder API. The differences are also highlighted in Table 5 which present the permeability and diffusion coefficients calculated according to Eqs. (2) and (3), respectively.

The permeability of the APIs and THEDES systems was calculated to be in the order of $10^{-5} \mathrm{~cm} / \mathrm{s}$, however significant differences may be observed between each pair API-THEDES. For example in the case of Ibu there is a 3 fold increase in permeability, whereas in the case of BA the increase is of 7.5 times. On the contrary, for the system with PA no significant differences were observed.

The diffusion coefficient is a parameter which relates the amount of API diffused with time. Hence, to a higher diffusion coefficient corresponds a faster diffusion of the API through a membrane. For all the systems studied an increase in the diffusion coefficient of the API when complexed in the THEDES form is observed (Table 5).

Taking into account the solubility results obtained it was expected that the diffusion of the API in the THEDES form would be faster than of the pure API, as the higher the solubility, the higher the driving force. For the case of Ibu and BA the high increase in solubility resulted in a high increase in the diffusion as well. Once again, in the case of the system menthol:PA differences in the diffusion coefficient and permeability are not as significant as for the other two APIs.

\subsection{Biopharmaceutics classification system}

The experimental data obtained in this study can be fit in the biopharmaceutics classification system plot of solubility, measured as the volume required to dissolve $1 \mathrm{mg}$ of API, versus permeability (Fig. 8).

According to the values described in the literature for the limits of the classification system, a drug is considered to be highly soluble when the volume required to dissolve $1 \mathrm{mg}$ is lower than $250 \mathrm{~mL}$. In which concerns the permeability, a highly permeable drug is one that has a permeability higher than $6 \times 10^{-6} \mathrm{~cm} / \mathrm{s}$. These were the values considered for the origin of Fig. 7. The different classes of drugs are also defined in each quadrant. The systems prepared present different behaviours, nonetheless overall an increase in both solubility and permeability is noticed when the API is in the THEDES form. Ibu, which in its native form falls in Class II type of drugs, has when in combination with menthol improved the solubility and permeability such that it can now be classified as a Class I type. BA, classified as Class III drug improves to a Class I system when in combination with menthol in a molar ratio of 3:1. PA, is per se a highly soluble and permeable drug and does not change its classification when in the THEDES form, on the contrary, as it is esterified, the THEDES system presents a low stability.

\section{Conclusions}

The THEDES system prepared, namely menthol:ibu (3:1); menthol:BA (3:1), menthol:PA (2:1) and menthol:PA (3:1) were characterized by NMR spectroscopy. The slope of the chemical shift of the hydrogen bonded protons with temperature, which reflects the activation energy of the hydrogen bond, correlated for all the THEDES in this work with an intermolecular hydrogen bond, with similar activation energies.

The transport properties of the mixture components in the THEDES were also investigated by PFG NMR spectroscopy, which showed to be independent of the molecular size of the carboxylic acid (API). The diffusion coefficient of the APIs were lower than of the menthol. A probable explanation is that the API forms dimers, which decreases their mobility in the global THEDES system. By combing the NMR diffusion and viscosity data, the diffusion mechanism shows a deviation from the classical theory, similar to the one observed in ionic liquids.

Regarding THEDES solubility and permeability behavior, it was demonstrated that the THEDES systems prepared present not only enhanced drug solubility in isotonic solution at $\mathrm{pH} 7.4$, but also

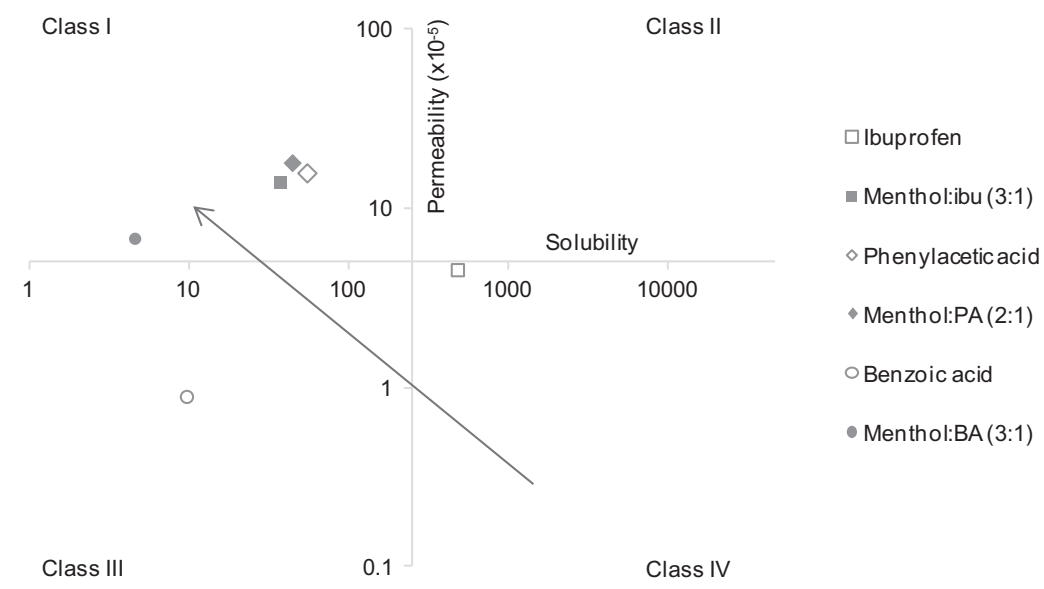

Fig. 8. Solubility versus permeability of both APIs (open symbols) and THEDES (closed symbols). Solubility in $\mathrm{mg} / \mathrm{mL}$ and permeability in $\mathrm{cm} \mathrm{s}{ }^{-1}$. 
present enhanced permeability. The manipulation of the physical state of the APIs, changing from solid form to liquid in the complex THEDES system has proven to be a simple and efficient methodology to improve the bioavailability of the APIs. In terms of biopharmaceutical classification this is translated into a change from class IV and III to class I systems, i.e. systems with high solubility and permeability. The experimental data obtained and presented in this work highlights the interest in deep eutectic systems as new formulations for improved therapeutics and the results presented open new perspectives in pharmaceutical engineering.

\section{Acknowledgments}

The research leading to these results has received funding from the European Union's Seventh Framework Programme (FP7/20072013) under grant agreement $n^{\circ}$ REGPOT-CT2012-316331POLARIS and from Project "Novel smart and biomimetic materials for innovative regenerative medicine approaches (Ref.: RL1 - ABMR - NORTE-01-0124-FEDER-000016)" co-financed by North Portugal Regional Operational Programme (ON.2 - O Novo Norte), under the National Strategic Reference Framework (NSRF), through the European Regional Development Fund (ERDF). Funding was also provided by Fundação para a Ciência e a Tecnologia, through contracts LAQV-REQUIMTE: UID/QUI/50006/2013 and UCIBIOREQUIMTE: UID/Multi/04378/2013. This work was co-financed by the ERDF under the PT2020 Partnership Agreement (POCI-010145-FEDER-007728).

\section{Appendix A. Supplementary material}

Supplementary data associated with this article can be found, in the online version, at http://dx.doi.org/10.1016/j.ejpb.2017.02.003.

\section{References}

[1] Y. Kawabata et al., Formulation design for poorly water-soluble drugs based on biopharmaceutics classification system: basic approaches and practical applications, Int. J. Pharm. 420 (1) (2011) 1-10.

[2] G.L. Amidon et al., A theoretical basis for a biopharmaceutic drug classification - the correlation of in-vitro drug product dissolution and in-vivo bioavailability, Pharm. Res. 12 (3) (1995) 413-420.

[3] C. Leuner, J. Dressman, Improving drug solubility for oral delivery using solid dispersions, Eur. J. Pharm. Biopharm. 50 (1) (2000) 47-60.

[4] R. Thakuria et al., Pharmaceutical cocrystals and poorly soluble drugs, Int. J. Pharm. 453 (1) (2013) 101-125.

[5] A. Martin, M.J. Cocero, Micronization processes with supercritical fluids: fundamentals and mechanisms, Adv. Drug Deliv. Rev. 60 (3) (2008) 339-350.

[6] P.B. Malafaya, G.A. Silva, R.L. Reis, Natural-origin polymers as carriers and scaffolds for biomolecules and cell delivery in tissue engineering applications, Adv. Drug Deliv. Rev. 59 (4-5) (2007) 207-233.

[7] P.B. Malafaya et al., Drug delivery therapies I - General trends and its importance on bone tissue engineering applications, Curr. Opin. Solid State Mater. Sci. 6 (4) (2002) 283-295.
[8] P.B. Malafaya et al., Drug delivery therapies II. Strategies for delivering bone regenerating factors, Curr. Opin. Solid State Mater. Sci. 6 (4) (2002) 297-312.

[9] S. Cherukuvada, A. Nangia, Eutectics as improved pharmaceutical materials: design, properties and characterization, Chem. Commun. 50 (8) (2014) 906923.

[10] I.M. Aroso, R. Craveiro, A. Rocha, M. Dionísio, S. Barreiros, R.L. Reis, A. Paiva, A. R.C. Duarte, Design of controlled release systems for THEDES - therapeutic deep eutectic solvents, using supercritical fluid technology, Int. J. Pharm. 492 (1-2) (2015) 73-79.

[11] P.W. Stott, A.C. Williams, B.W. Barry, Transdermal delivery from eutectic systems: enhanced permeation of a model drug, ibuprofen, J. Control. Release $50(1-3)(1998)$ 297-308.

[12] H.G. Morrison, C.C. Sun, S. Neervannan, Characterization of thermal behavior of deep eutectic solvents and their potential as drug solubilization vehicles, Int. J. Pharm. 378 (1-2) (2009) 136-139.

[13] H. Wang et al., Simultaneous membrane transport of two active pharmaceutical ingredients by charge assisted hydrogen bond complex formation, Chem. Sci. 5 (9) (2014) 3449-3456.

[14] A. Paiva et al., Natural deep eutectic solvents - solvents for the 21st Century, Acs Sustain. Chem. Eng. 2 (5) (2014) 1063-1071.

[15] N. Schultheiss, A. Newman, Pharmaceutical cocrystals and their physicochemical properties, Cryst. Growth Des. 9 (6) (2009) 2950-2967.

[16] I.M. Aroso et al., Dissolution enhancement of active pharmaceutical ingredients by therapeutic deep eutectic systems, Eur. J. Pharm. Biopharm. 98 (2016) 57-66.

[17] A.K. Jain, N.S. Thomas, R. Panchagnula, Transdermal drug delivery of imipramine hydrochloride. I. Effect of terpenes, J. Control. Release 79 (1-3) (2002) 93-101.

[18] D.H. Wu, A.D. Chen, C.S. Johnson, An improved diffusion-ordered spectroscopy experiment incorporating bipolar-gradient pulses, J. Magn. Reson., Ser. A 115 (2) (1995) 260-264.

[19] E.O. Stejskal, J.E. Tanner, Spin diffusion measurements: spin echoes in the presence of a time-dependent field gradient, J. Chem. Phys. 42 (1) (1965) 288 292.

[20] J.M. Silva et al., Tailored freestanding multi layered membranes based on chitosan and alginate, Biomacromolecules 15 (10) (2014) 3817-3826.

[21] H.Y. He, X. Cao, L.J. Lee, Design of a novel hydrogel-based intelligent system for controlled drug release, J. Control. Release 95 (3) (2004) 391-402.

[22] L.Y. Chu et al., Control of pore size and permeability of a glucose-responsive gating membrane for insulin delivery. J. Control. Release 97 (1) (2004) 43-53.

[23] P.H. Che et al., Hydrogen bond distinction and activation upon catalytic etherification of hydroxyl compounds, Chem. Commun. 51 (6) (2015) 1077 1080.

[24] P. Charisiadis et al., ${ }_{1} \mathrm{H}$ NMR as a structural and analytical tool of intra- and intermolecular hydrogen bonds of phenol-containing natural products and model compounds, Molecules 19 (9) (2014) 13643-13682.

[25] N. Sewald, H.D. Jakubke, Peptides: Chemistry and Biology, Wiley, 2015.

[26] Y. Li et al., An insight into the extraction of transition metal ions by picolinamides associated with intramolecular hydrogen bonding and rotational isomerization, RSC Adv. 4 (56) (2014) 29702-29714.

[27] C. D’Agostino et al., Molecular motion and ion diffusion in choline chloride based deep eutectic solvents studied by ${ }_{1} \mathrm{H}$ pulsed field gradient NMR spectroscopy, Phys. Chem. Chem. Phys. 13 (48) (2011) 21383-21391.

[28] C. Florindo et al., Insights into the synthesis and properties of deep eutectic solvents based on cholinium chloride and carboxylic acids, Acs Sustain. Chem. Eng. 2 (10) (2014) 2416-2425.

[29] S.F. Ng et al., Validation of a static Franz diffusion cell system for in vitro permeation studies, Aaps Pharmscitech 11 (3) (2010) 1432-1441.

[30] S.F. Ng et al., A comparative study of transmembrane diffusion and permeation of ibuprofen across synthetic membranes using Franz diffusion cells, Pharmaceutics 2 (2010) 209-223. 